\title{
ABSTRACTS OF CURRENT LITERATURE
}

\section{JOURNAL OF THE AMERICAN MOSQUITO CONTROL ASSOCIATION}

\section{Ability of Essential Oil Candles to Repel Biting Insects in High and Low Biting Pressure Environments}

Mosquitoes are endemic vectors for illness as their potential breeding sites increase exponentially with urbanization. ${ }^{1}$ DEET (N,N-diethyl-3-methylbenzamide), the leading skin-applied repellent, repels a broad spectrum of biting insects. However, it is not as appealing as natural substances, and it loses efficacy with perspiration and mechanical debridement. ${ }^{2,3} \mathrm{Ad}-$ ditionally, with increasing concentrations and excessive applications, DEET can cause skin and mucous membrane irritation, headache, nausea, dizziness, disorientation, and seizures. ${ }^{2,4}$

This study evaluated the efficacy of 3 types of essential oil candles to repel mosquitoes. All candles were $85 \mathrm{~g}$, containing $5 \%$ essential oil of citronella, linalool, or geraniol. A paraffin candle of the same weight without any repellent or fragrance was used as a control. Outcome measures included mosquito biting and mosquito concentration.

Four candles of each type were arranged in squares of $1 \mathrm{~m}$, $2 \mathrm{~m}$, or $3 \mathrm{~m}$ on each side, with a Centers for Disease Control and Prevention-lighted trap (without $\mathrm{CO}_{2}$ ) placed in the middle. The experiments were repeated over 10 nights, with the trap operational for 8 hours between 2000 hours and 0400 hours. A total of 1637 female mosquitoes and 870 female sand flies were caught in the traps. Compared with the control, geraniol candles were the most effective, decreasing the female mosquito load by $82 \%$ within $1 \mathrm{~m}$ and $50 \%$ at $3 \mathrm{~m}$. Linalool decreased the load by $65 \%$ at $1 \mathrm{~m}$ and $36 \%$ at $3 \mathrm{~m}$. Citronella candles were the least effective, reducing female mosquito concentration by $35 \%$ at $1 \mathrm{~m}$ and $12 \%$ at $3 \mathrm{~m}$. Similar results in sand fly reduction were found.

Next, comparing only the geraniol candles with paraffin control candles, 6 volunteers ( 3 male, 3 female) were used to evaluate a reduction in actual bites. This trial was repeated for 6 nights total. Each trial alternated subject and candle combinations to avoid sex and locational bias. The trial test period was 1 hour, from $2000 \mathrm{~h}$ to $2100 \mathrm{~h}$. Mosquito landing, probing, and biting on the subject's left arm and hand were counted and recorded on data sheets in intervals of 5 minutes. Immediately before each trial, the exposed skin on each volunteer was cleaned with $70 \%$ isopropyl alcohol. The volunteers avoided alcohol, caffeine, and fragrance products (eg, perfume, cologne, hair spray, lotion) during the entire test period of 6 nights. The geraniol candle was tested in high biting- and low biting-pressure environments. In these zones, unprotected subjects (paraffin control) received an average of 19 and 8 mosquito interactions per 5 minutes, respectively. Geraniol candles reduced mosquito-human interaction by $56 \%$ in the high biting-pressure zone and by $62 \%$ in the low biting-pressure zone.

In conclusion, of the 3 candles initially tested, geraniol candles repelled insects most effectively, followed by linalool and then citronella. Within $1 \mathrm{~m}$, geraniol candles reduced total mosquito concentration by $82 \%$ and mosquito landing, probing, and biting by an average of $59 \%$ compared with a paraffin control. Thus, geraniol still allowed sufficient contacts for bites to occur. The efficacy of botanical candles dropped significantly with distance from the candles extending beyond $1 \mathrm{~m}$. Botanical candles offer modest protection from biting insects and should be used in conjunction with other repellents for appropriate disease control and prevention.

(J Am Mosquito Control Assoc. 2008;24:154-160) GC Müller, A Junnila, VD Kravchenko, EE Revay, J Butler, OB Orlova, RW Weiss, and Y Schlein.

Prepared by Jeremiah Ray, MS3 Stanford School of Medicine, Stanford, CA, USA

\section{References}

1. Lambdin B, Schmaedick MA, Burkot TR. Utilization of domestic and natural containers by Aedes oceanicus in American Samoa. J Med Entomol. 2008;45:758-762.

2. Carroll JF, Solberg VB, Klun JA, Kramer M, Debboun M. Comparative activity of deet and AI3-37220 repellents against the tick Ixodes scapularis and Amblyomma americanum (Acari: Ixodidae) in laboratory bioassays. J Med Entomol. 2003;41:249-254.

3. Fradin MS, Day JF. Comparative efficacy of insect repellents against mosquito bites. N Engl J Med. 2002;347:1318.

4. Bell JW, Veltri JC, Page BC. Human Exposures to N,Ndiethyl-m-toluamide insect repellents reported to the American Association of Poison Control Centers 1993-1997. Int J Toxicol. 2002;21:341-352.

\section{BRITISH MEDICAL JOURNAL}

\section{Imported Malaria and High Risk Groups: Observational Study Using UK Surveillance Data 1987-2006}

Malaria acquired by travelers to endemic regions represents a burden of disease largely preventable through chemoprophylaxis and antimosquito measures. Accurate risk stratification of travelers would also aid in malaria prevention. This study aimed to assist physicians in the risk assessment of travelers by analyzing trends and demographics of infected individuals. 
This observational study used prospectively gathered data on malaria presenting in the UK. These data were gathered by the National Malaria Reference Laboratory Surveillance program between 1987 and 2006. Malaria surveillance in the UK is a passive detection process that relies on statutory notification by local authorities and clinicians. Only cases with parasitologic confirmation by either blood films or tissue histology were included. Data collected from each case included date of birth, sex, country of birth, date of arrival in UK, country or region visited, purpose of travel, duration of travel, use of prophylaxis, and details of illness (ie, date of onset, date of treatment, and method of diagnosis). Supplementary information came from death certificates and postmortem findings for all malaria-associated deaths.

This study included 39000 cases of malaria reported in the UK. Of those, 34359 cases, or $88 \%$, had a reported travel history. Malaria due to Plasmodium faciparum increased over the course of the study period $(P<.0001)$, while cases of Plasmodium vivax malaria decreased $(P<.0001)$. A higher frequency of malaria was seen in travelers whose stated purpose of travel was to visit friends and family $(65 \%, 95 \% \mathrm{CI}$ $64 \%-65 \%$ ). In cases with a reported travel history, $72 \%$ occurred after travel to Africa and $25 \%$ occurred after travel to South Asia. Use of chemoprophylaxis among cases was lower in travelers whose stated purpose of travel was to visit family in comparison with travelers with other stated purposes of travel (Mantel-Haenszel odds ratio adjusted for age and sex = $0.23,95 \%$ CI $0.20-0.25$ ).

Despite its limitations, this study identified that travelers who are visiting family abroad demonstrate a higher frequency of malaria and might benefit from increased prophylaxis. This is an observational study that relies on passive notification by local authorities and clinicians, and so the prevalence of imported malaria may be higher than in the actual population due to underreporting. Also, reporting physicians did not report full details on travel history and prophylaxis. The study does not include information on patients' prior exposure to malaria, type of chemoprophylaxis used, or type of antimosquito techniques utilized. However, this study does highlight the importance of health care providers' tailoring malaria prevention strategies to patients from migrant families traveling to endemic regions.

(BMJ. 2008;337:1-7) AD Smith, DJ Bradley, V Smith, M Blaze, RH Behrens, PL Chiodini, and CJ Whitty.

Prepared by Chioma Agbo, MS4 Stanford Medical School, Stanford, CA, USA

\section{SOUTHERN MEDICAL JOURNAL}

\section{River Tree Rope Swing Injuries}

This study was a retrospective analysis of injuries that occurred during the use of river tree rope swings (RTRS) between 2002 and 2006. Injury reports were derived from the National Electronic Injury Surveillance System, US legal literature, and news reports. As a supplement to the discussion, the authors visited and evaluated 15 existing RTRS in Mississippi, Tennessee, Missouri, and North Carolina. This small group of existing swings was not prospectively observed for injury and only assessed for safety. Additional safety assessments were made of retrieval lines, which are small-diameter ropes used to return the primary swing to shore. This was done by simulating an entangled leg with a wooden rod and recording damage to the rod caused by return with a retrieval line.

Seventy-three patients between the ages of 5 and 25 years with RTRS injuries were identified. Lower extremity injuries were the most common, occurring in $38 \%$ of National Electronic Injury Surveillance System cases. Finger fractures were the single most common injury, accounting for $22 \%$ of all cases. Six deaths were reported, of which 4 resulted from drowning, 1 from head trauma, and 1 from multisystem trauma. Field observation revealed other hazards, such as poison ivy, rusty nails, broken bottles, water depth as low as 18 inches, and fragile ropes. Also, small-diameter retrieval lines led to increased injury, primarily through abrasion.

This study was limited by its size and sources of data, and as acknowledged by the authors, it likely underestimated the injuries related to rope swings by a significant degree. Nevertheless, it did point out many risks associated with RTRS and can be used for educating the public, raising awareness, and modifying swings. Although the distance from RTRS to emergency care was not published, the authors suggested that the remote location of many RTRS makes them difficult to monitor and may delay emergency response. This study underscored the fact that RTRS are not always a benign pastime and that improved education about potential hazards and possible injuries that can occur need to be made clearer to the public. It is obvious that the use of RTRS will continue. However, parents and adolescents should be able to swim, remove swings from shallow water, and help monitor the condition of existing rope swings with safety in mind.

(South Med J. 2008;14:699-702) WH Sorey, LD Cassidy, J Crout, and P Blount.

Prepared by Jan Vobecky, MD Stanford-Kaiser Emergency Medicine Residency, Stanford, CA, USA

\section{BRITISH JOURNAL OF SPORTS MEDICINE}

\section{Preventive Effect of Pre-Dive Hydration on Bubble Formation in Divers}

This small prospective crossover cohort study evaluated the effects of hydration on intravascular bubble formation after decompression. It assumed that intravascular bubble production directly correlates to the same spectrum of disease as decompression sickness. Previous data, summarized in this study, provided conflicting evidence relating to the benefit of hydration in animal models of decompression. The study is unique in using human subjects.

Eight military divers participated in this 1-week trial. Each 
person dived twice, once without hydration for at least 90 minutes before the dive and once with oral hydration totaling 1300 $\mathrm{mL}$ of 324 mOsm saline-glucose beverage beginning $90 \mathrm{~min}-$ utes before diving. These dives were separated by 2 days, with half the group hydrating on the first dive and the other half going without hydration on their first dive. On their second dive, each participant changed to the alternate group. After a 30-minute, 30-m dive, the decompression procedure followed standard French Navy protocol, allowing for a 9-minute stop at $3 \mathrm{~m}$. The investigators measured bubble activity at standard intervals over a 90-minute period after the dive with Doppler ultrasound. They graded the signal from the bubbles and converted them to a Kissman integrated severity score, which allowed for quantification of bubble activity.

Doppler ultrasound demonstrated decreased bubble production in the hydrated patients by way of a statistically significant decrease in mean Kissman score from 19 to 4 . None of the divers suffered from decompression sickness. Although limited by its small sample size, this trial is well designed to direct larger in vivo studies to better evaluate the potential protective affect of predive hydration on bubble formation. Furthermore, the connection between bubble formation as assessed by Kissman score and development of decompression sickness has not been established, although it may serve as a surrogate marker and needs to be further evaluated in animal studies.

(Br J Sports Med. 2008;4:1-11) E Gempp, JE Blatteau, JM Pontier, C Balestra, and P Louge.

Prepared by Anil Menon, MD Stanford-Kaiser Emergency Medicine Residency, Stanford, CA, USA.

\section{INTERNATIONAL SOCIETY OF TRAVEL MEDICINE}

\section{Environmental Hazards in Nepal: Altitude Illness, Environmental Exposures, Injuries, and Bites in Travelers and Expatriates}

Not surprisingly, travelers to foreign countries encounter a different spectrum of disease from their country of origin. Trauma, for example, increases to a leading cause of death among travelers, accounting for $25 \%$ of such deaths. ${ }^{1}$ Travelers to a foreign country, such as Nepal, face a potentially greater shift in the character of disease they face due to the more dramatic changes in geography and altitude. More than 300000 people each year venture to Nepal from foreign countries and present with illnesses related to not only the altitude but also the activities they undertake and the flora and fauna they contact. It was the aim of this study to assess these illnesses and risk factors affecting foreigners in Nepal.

When these travelers and their fellow expatriates do contract an illness, they will most likely present to the Canadian International Water and Energy Consultants clinic. This clinic is located in Kathmandu and serves as the primary source of medical care for travelers and expatriates, usually from the United States, Canada, Western Europe, Japan, Australia, and New Zealand. This study is unique in using historical data recorded at the clinic to infer relationships between foreigners' demographics and illnesses in this region.

The study's database spanned 7 years, contained 15462 patients, and excluded immigrants and indigenous persons. Foreigners were segregated into travelers and expatriates (the duration of time living in Nepal necessary to become an expatriate was not clearly defined). The purpose of travel was categorized as tourism, business, education, volunteering, and visiting relatives or friends. Among other categories, an illness could be diagnosed as an environmental illness. An environmental illness could include lacerations, fractures, sprains, altitude illness, bites, dehydration, frostbite, heat exhaustion, and the like.

The results revealed that environmental diagnoses were more common in travelers than in expatriates, $17 \%$ vs $9 \%$ respectively, as was altitude illness, $4.7 \%$ vs $0.1 \%$. Those travelers who suffered from an environmental- or exposure-related illness were slightly less likely to have received pretravel advice, with statistically significant odds ratios of 0.8 to 0.9 . The authors inferred that pretravel advice could target and prevent injury in tourists. Although medical advice is likely beneficial before any trip, it still cannot be directly correlated to injury prevention through this study. Tourists were likely engaged in more injury-prone activities than expatriates, and those people who did not seek advice might be prone to more risky activities. Also, as the study notes, there was no indication as to the antecedent activity, which might help further determine the utility of specific travel advice. It was noted that expatriates were more likely to be bitten by dogs and travelers were more commonly bitten by monkeys. Although the authors assume that this outcome was probably activity related, it might help steer advice and possible prophylaxis.

(J Travel Med. 2007;14:361-368) AK Boggild, C Costiniuk, KC Kain, and P Pandey.

Prepared by Anil Menon, MD Stanford-Kaiser Emergency Medicine Residency, Stanford, CA, USA

\section{Reference}

1. Hargarten SW, Baker TD, Guptill K. Overseas fatalities of United States citizen travelers: an analysis of deaths related to international travel. Ann Emerg Med. 1991;20:622-626. 